\title{
CTF3 PROTOTYPES: DESIGN, TESTS AND MEASUREMENTS
}

\author{
A. Ghigo, D. Alesini, C. Biscari, R. Boni, A. Clozza, G. Delle Monache, A. Drago, A. Gallo, \\ F. Marcellini, C. Milardi, C. Sanelli, M. Serio, F. Sgamma A. Stecchi, A. Stella, M. Zobov, \\ INFN-LNF, Frascati, Italy. \\ R. Corsini, L. Rinolfi, CERN, Geneva, Switzerland.
}

\section{Abstract}

The high efficiency in the $30 \mathrm{GHz}$ power production is maintained if the beam quality is preserved along the rings and the transfer lines of the CLIC Test Facility (CTF3) compressor system [1,2]. The energy loss and energy spread, caused by vacuum chamber components coupling impedance and by coherent synchrotron radiation (CSR) emission, must be minimized.

The magnetic layouts of the first ring of the compressor system, called Delay Loop (DL) and of the transfer line between the Linac and DL are presented. Tests and measurements on several vacuum chamber components prototypes are reported. The $3 \mathrm{GHz} \mathrm{RF}$ deflectors, that will provide the injection in the Combiner Ring, have been studied, realized, tested, installed and successfully used in the first interlaced bunch trains injection in the CTF3 preliminary phase. A standing wave $1.5 \mathrm{GHz}$ RF deflector for injection into the Delay Loop is proposed.

\section{INTRODUCTION}

The $30 \mathrm{GHz}$ RF power production, needed in the CLIC project, depends on the drive beam structure; after the recombination system very short bunches with regular spacing are required. To preserve the beam quality in the CTF3 compressor system the ring lattices are isochronous and the $\mathrm{R}_{56}$ of the transfer lines is kept very small.

The study of rings and transfer lines optics has been reported in the CTF3 Design Report [1] and in several articles [2,3]. The coherent synchrotron radiation effect for short bunches as well as the vacuum chamber coupling impedance become important issues, leading to an energy loss and to an increase in energy spread. In fact, the energy losses give rise to relative phase errors between bunches through non perfect isochronicity, which results in deterioration of the timing between individual bunches and merging trains; the energy spread leads to bunch lengthening and phase space distortion.

The INFN Frascati laboratories will provide the first of the two rings of the compressor system called Delay Loop (DL). At the exit of this ring the frequency of the bunches coming from the Linac $(1.5 \mathrm{GHz})$ will be doubled with an increment of the current by a factor two. A magnetic bunch stretcher chicane is part of the transfer line that join the Linac with the DL; it is used to increase the bunch length in order to reduce CSR and impedance effects.

Therefore the magnetic stretcher/compressor chicane was designed with an $\mathrm{R}_{56}$ tunable in the range of $+50,-30$ $\mathrm{cm}$ that allows to vary the bunch length in a very wide range.

Systematic experimental studies of the CSR effect in the compressor are also foreseen with an energy analyser and an emittance measurement system at the chicane exit. In this paper the study and the prototype realization of several components of the vacuum chamber are described. The tests of the RF deflectors realized by LNF, and successfully used in the CTF3 preliminary phase [5], are also reported.

\section{MAGNETIC LAYOUT}

The optics of Delay Loop and Transfer lines have been well studied including multiparticle tracking [3]. The magnetic layout of the Delay Loop and transfer lines has been completed using existing dipole and quadrupole magnets of the EPA complex in CERN together with the injection extraction septa magnet. In Fig. 1 the layout of the magnetic elements is shown.

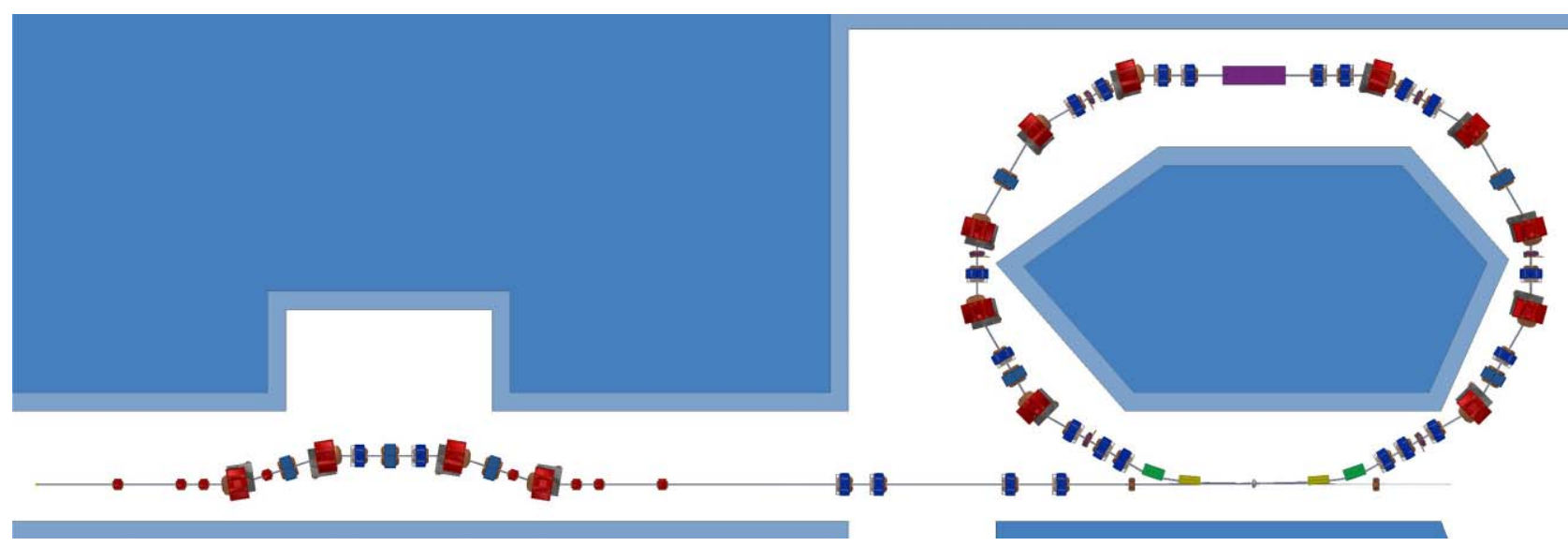

Figure 1: Layout of the Delay Loop and Transfer Line magnetic elements. 


\section{PROTOTYPES}

The first prototypes vacuum chamber components studied and realized are the pump ports, shielded bellows and beam position monitors. The longitudinal impedance measurements of these devices have been done because their large number along the rings and transfer lines can contribute in significant way to the impedance. The Combiner Ring RF deflectors have been also tested and installed in the CTF3 preliminary phase [5]. The Delay Loop RF deflector has been studied.

\section{Vacuum Pump Port}

In the design of vacuum pump ports RF screen the hidden slots idea, applied successfully for PEP II, has been adopted. This allows to reduce by some orders of magnitude the coupling impedance and to prevent radiation into the pump chamber. The realized RF screen consists of 38 grooves $72 \mathrm{~mm}$ long, $2 \mathrm{~mm}$ wide and $2 \mathrm{~mm}$ deep in the rectangular vacuum chamber pipe with $4 \mathrm{~mm}$ thickness; these grooves are used to decrease the coupling impedance. Along each groove 25 circular holes of $1 \mathrm{~mm}$ (smaller than the bunch longitudinal size) are drilled for pumping purpose; these holes prevent penetration of the RF fields outside of the beam pipe. To decrease further the impedance in the rectangular geometry of the beam pipe, the grooves are placed closer to the chamber corners and there are no grooves on the lower and upper desks of the chamber near the symmetry axis.

The impedance of the prototype section, $8 \mathrm{~cm}$ long, that provides a vacuum conductance of $60 \mathrm{l} / \mathrm{s}$, has been measured with the wire methods with resistive matching. The measurements, extended up to $4.5 \mathrm{GHz}$ (maximum allowable range with resistive matching), have shown that the impedance values are too small to be detected by the measurement apparatus. The calculated impedance of these pumping screen is $\mathrm{Z} / \mathrm{n}=\mathrm{j} 4.3^{*} 10^{-6} \Omega$. No resonant mode seems to appear.

\section{Shielded Bellows}

A prototype of the shielded bellows that provides the vacuum chamber flexibility has been developed. The RF shielding is a sliding finger structure, similar to that used in the KEKB rings, with the same inner shape of the rectangular vacuum chamber. The number and the shape of the sliding contact have been chosen in order to reduce the coupling of the bunch field to the bellows structure; the thickness and position of the spring finger have been chosen to maximize the electrical contact. The bellows impedance, predominantly inductive, has been evaluated to be about $10^{-6} \Omega$.

\section{Beam Position Monitor}

The CTF3 BPM works in principle as a transformer excited by the beam. The beam image current is diverted to four metallic strips placed outside the vacuum chamber ceramic gap and short-circuited to the two ends of the metallic vacuum chamber. Small ferrite core transformers, placed at the end of each strip, provide signals whose amplitude depends on the beam position [1].

The striplines are surrounded by ferrite tiles to improve the low frequency response. Such ferrites, at high frequency, have high RF losses and damp unwanted resonances of the external metallic shield.

Measurements based on the coaxial wire method have been performed to estimate the transfer impedance of the device and showed a low frequency cut-off at $400 \mathrm{kHz}$ [3].

To investigate position sensitivities of the device, a series of measurements with a movable wire has been performed at the calibration bench shown in Fig.2.

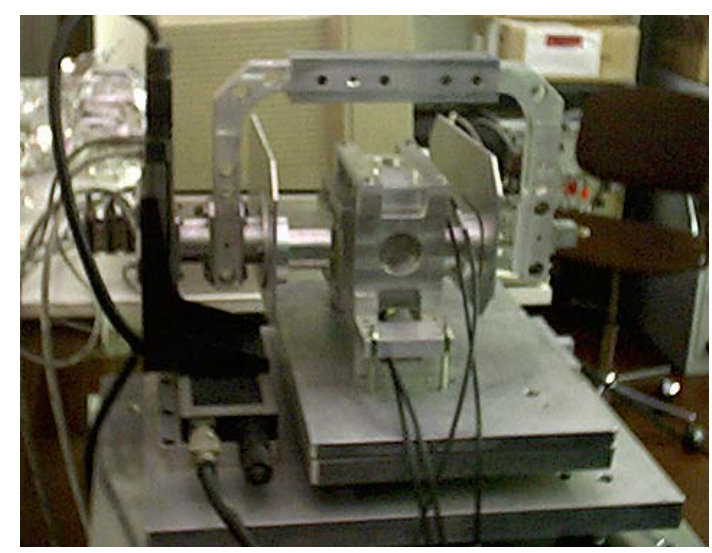

Figure 2: BPM calibration bench

Beam position is derived from the ratios $U, V$ (pseudopositions) between the peak intensities $v_{i}$ of the signals from the windings by using a non linear fit:

$$
x=k_{x} \cdot U+f(U, V) \quad y=k_{y} \cdot V+g(U, V)
$$

where $k_{\mathrm{x}}$ and $k_{\mathrm{y}}$ are the inverse sensitivities to beam displacement and $f(U, V)$ and $g(U, V)$ are polynomial functions.

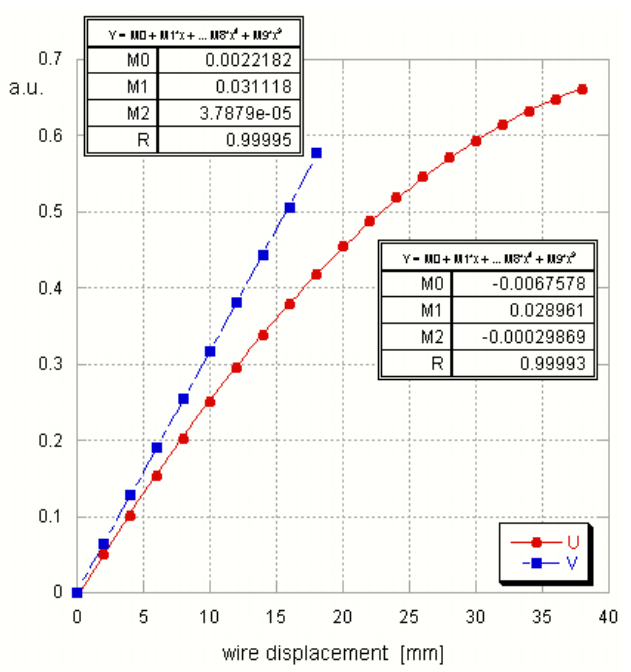

Figures 3: Position sensitivities along the horizontal and vertical axis 
The outputs $v_{i}$ at a fixed frequency from each secondary winding have been acquired for several transverse wire positions to derive coefficients of the polynomial functions $(f$ and $g$ ) used to correct non linearities of the BPM for large beam offsets (see Fig. 3).

Data from these measurements showed $20 \mathrm{~dB}$ maximum amplitude excursion between the four signals $V_{1}$ for a beam offset by $70 \%$ of the vacuum chamber dimensions.

In order to reduce the beam coupling impedance, the internal wall of the ceramic gap will be coated with a conductive film whit thickness that allows only lower frequency components of beam fields to propagate outside to be picked up by the secondary windings.

\section{RF DEFLECTORS}

\section{Combiner Ring RF Deflectors}

The bunch trains interlacing scheme of CTF3 is based on the use of RF deflectors as injection kickers. The Combiner Ring RF deflector design has been based on an already optimized TW deflecting structure. The interaction between the beam and the deflector operating mode (beam loading) has been studied to evaluate the impact on the quality of the extracted beam. We came to the conclusion that the beam loading in a pair of 10-cells TW deflectors does not produce a significant degradation of the beam quality, provided that the betatron phase advance between the two devices is properly chosen [1]. Following these results, two deflectors used in CTF3 preliminary and nominal phases have been built in collaboration with the Soltan Institute for Nuclear Studies (Swierk, Poland).

The INFN-LNF CTF3 group collaborated in the CTF3 preliminary phase (which started in autumn 2001 and has been completed during year 2002) having a goal to demonstrate the "funnelling" injection scheme and bunch train compression in an isochronous lattice. The main difference between these preliminary experiments and the nominal CTF3 is the limited beam current available from CERN LEP Injector Linac.

In CTF3 preliminary phase the concept of bunch train combination by means of RF deflectors has been demonstrated experimentally [5].

\section{Delay Loop RF Deflector}

The Linac bunch train, $1.4 \mu$ s long, is composed by sequence of $140 \mathrm{~ns}$ bunch trains, with $1.5 \mathrm{GHz}$ repetition rate, phase shifted by one $3 \mathrm{GHz}$ RF cycle. The odd trains are injected into the Delay Loop and recombined at the extraction after one turn with the pair incoming trains. This scheme is used to double the current in the $140 \mathrm{~ns}$ train increasing to $3 \mathrm{GHz}$ the bunch repetition rate. An RF deflector, working at $1.5 \mathrm{GHz} \mathrm{RF}$ frequency, placed at the conjunction point of transfer line and Delay Loop, provides the injection/extraction in/from the Delay Loop.

The pulsed RF source is a $1.5 \mathrm{GHz}$ Klystron, custom developed for this purpose, with maximum RF delivered power of $20 \mathrm{MW}$ with $5 \mu$ s pulse length. The requested deflection angle is $15 \mathrm{mrad}$ at the maximum energy of
$300 \mathrm{MeV}$ (nominal energy of the full current beam is 150 $\mathrm{MeV})$.

Different design solutions for this device have been investigated. A standing wave cavity, that has more compact design with respect to the multicell travelling wave structure and provides better kick efficiency, has been chosen. In Table 1 the characteristics of two different standing wave single-cell deflectors with different $\mathrm{Q}$ value are presented. Because of the filling time and finite RF pulselength, there are different deflection angles from head to tail of the bunch train coming from the Linac. The cavity with lower Q is less efficient but minimizes these kick differences that produce a transverse emittance growth.

Table 1. Different Q value cavities comparison

\begin{tabular}{|c|c|c|}
\hline $\mathbf{Q}$ & $\mathbf{4 2 0 0}$ & $\mathbf{5 8 0 0}$ \\
\hline $\mathrm{P}(15 \mathrm{mrad} @ 300 \mathrm{MeV})$ & $18 \mathrm{MW}$ & $14 \mathrm{MW}$ \\
\hline$\Delta \mathrm{V}$ start to end & $1.5 \%$ & $4.0 \%$ \\
\hline
\end{tabular}

Figure 4 shows the HFSS model of the developed single cell standing wave deflecting cavity. The cavity is fed by two waveguide placed on opposite sides in the horizontal plane.

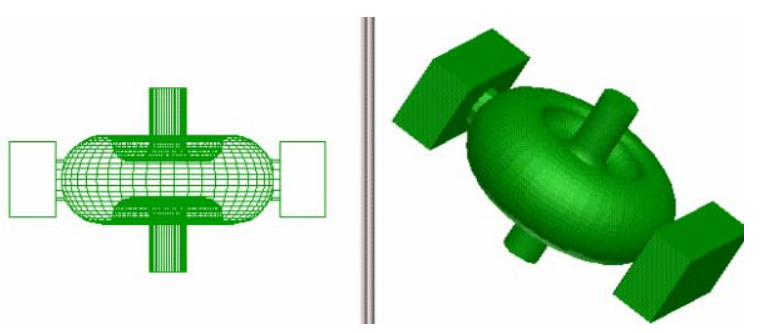

Figure 4: $1.5 \mathrm{GHz}$ RF deflector model

\section{ACKNOWLEDGMENTS}

The authors wish to thank G. Fontana, V. Lollo, and A. Zolla for the machine layout and the mechanical project of the presented prototypes; S. Pella for the technical support during measurements; the CTF3 CERN technical staff for the RF deflectors installation and power test.

\section{REFERENCES}

[1] G. Geschonke, A. Ghigo et al., "CTF3 Design Report” CERN/PS 2002-008 (RF), LNF-02/008 (IR)

[2] C. Biscari et al., "CTF3: Design of Driving Beam Combiner Ring”, EPAC'2000, Vienna, p.450.

[3] D. Alesini et al., "CTF3 Compressor System", Proceedings of EPAC 2002, Paris, France, p.416.

[4] D. Alesini et al., "RF Beam Deflector for CTF3 Combiner Ring", Proceedings of EPAC 2002, Paris, p. 2115 .

[5] F. Tecker et al. "Bunch Frequency Multiplication in the CLIC Test Facility CTF3" PAC 2003, these proceedings. 